\title{
Edmodo como herramienta virtual de aprendizaje
}

\section{Edmodo as a virtual learning tool}

Jorge Enrique Díaz Pinzón

Universidad Nacional de Colombia., Colombia

Autor para correspondencia: jediazp@unal.edu.co,jorgediaz333@gmail.com

Fecha de recepción: 11 de Mayo de 2017 - Fecha de aceptación: 30 de Septiembre de 2017

Resumen: En la actualidad nos ubicamos en un proceso de constantes cambios y transformaciones en la que surge la necesidad de la incorporación de nuevas tecnologías de la información y la comunicación en diversos aspectos del conocimiento, incluidos en el aspecto educativo. Por tal motivo la experiencia de investigación, de metodología cualitativa y cuantitativa, indago sobre el uso de Edmodo como una herramienta virtual de aprendizaje de la estadística descriptiva para el grado once de la Institución Educativa General Santander. Para tal fin de la investigación se utilizaron encuestas directas de carácter individual y de acuerdo a su forma de tipo cerrada. Estás fueron aplicadas como una técnica que utiliza un cuestionario con diversos tipos de preguntas con el fin de conseguir mediciones cuantitativas sobre algunas características objetivas y subjetivas de la población, que después ser sistematizadas, analizadas e interpretadas se observó por parte de los estudiantes como la implementación del aula virtual edmodo sirvió como una herramienta virtual de aprendizaje y de este modo colocar a los estudiantes de grado once en contacto con las nuevas tecnologías de la información y la comunicación -TIC- relacionados con los estándares de la estadística descriptiva.

Palabras claves: edmodo; aula virtual; estadística descriptiva

\begin{abstract}
Currently we are in a process of constant changes and transformations in which the need for incorporating new information technologies and communication in various aspects of knowledge, including in the educational aspect arises.

According to UNESCO (2005) education systems worldwide are currently facing the challenge of using new information technology in communication and ICT to provide its students with the tools and knowledge necessary for the XXI century.

Therefore the research experience of qualitative and quantitative methodology, I investigate about using Edmodo as a virtual learning tool descriptive statistics for the juniors of School General Santander.To this end research direct survey of individual character and according to their way of closed type is used. You are were applied as a technique using a questionnaire with different types of questions in order to get quantitative measurements on some objective and subjective characteristics of the population, which then be systematized, analyzed and interpreted was seen by students as the implementation virtual Classroom Edmodo served as a virtual learning tool and thus placing eleventh grade students in touch with the new technologies of information and communication -ICT- as the computer and the Internet and deepen related content standards of descriptive statistics.
\end{abstract}

Key words: edmodo; virtual classroom; descriptive statistics 


\section{Introducción}

El presente trabajo de investigación trata de un aspecto importante en el campo educativo, pues se trata de la implementación de un aula virtual, EDMODO, como herramienta virtual de aprendizaje; dónde los estudiantes de grado once de la I.E. General Santander podrán complementar las actividades realizadas en clase específicamente en la asignatura estadística descriptiva.

EDMODO permite establecer un espacio virtual de comunicación con los estudiantes y docentes, en el que se pueden hacer comentarios y aportes de las actividades realizadas, adjuntar archivos y enlaces, establecer un calendario de trabajo, así como de actividades, evaluaciones y gestionarlas.

Entre las prerrogativas de este servicio hay que matizar que es gratuito, está en español y que pueden registrarse los menores de edad. Asimismo, y esto es bien importante, la página es privada de forma predeterminada, lo que significa que la información sólo es accesible para los estudiantes que ingresan con su nombre de usuario y contraseña, es decir, el estudiante y profesor registrado.

Es de resaltar la importancia de la necesidad de implementar un aula virtual para mejorar las habilidades y capacidades en ambientes virtuales de aprendizaje en los estudiantes como un elemento fundamental en el proceso de integración con las tecnologías de la información y comunicación Tics y de formación académica en la educación.

Asimismo el aporte de esta investigación es el fomentar el uso de EDMODO en todas las áreas de conocimiento en la institución educativa, y de esta forma poder interactuar y recibir aportes de los demás docentes y estudiantes acerca de las bondades y expectativas de esta investigación en el quehacer diario de la comunidad educativa General Santander.

\section{Formulación del problema}

\section{Planteamiento de estudio}

¿Cómo puede la plataforma Edmodo contribuir en la enseñanza de la estadística descriptiva a los estudiantes de la I.E. General Santander de Soacha-Cundinamarca? La mayoría de docentes Institución Educativa General Santander, no cuentan con una alternativa extra- aula para desarrollar procesos educativos con miras a generar aprendizajes significativos que sirvan de modelo al resto de la comunidad educativa.

"El uso generalizado de las Tics en los procesos de aprendizaje se constituye en una "condición necesaria pero no suficiente" para la consecución de un éxito en el proceso y ha generado cambios en el paradigma educativo tradicional que tenía como centro a la figura del profesor, poseedor del conocimiento que trasmite a sus alumnos, a un paradigma innovador que centra la atención en el estudiante como gestor de sus procesos de aprendizaje, en donde el profesor pasa a ser actor de motivación, de guía y asesoría, de supervisión y evaluación.”(Pacheco., 2010) 
Las oportunidades que ofrece la formación con apoyos virtuales en el campo de la estadística son notorios en hechos tales como: flexibilización de los horarios, personalización y autogestión de algunos contenidos y actividades del curso, desarrollo de destrezas tecnológicas, entre las cuales las comunicativas a través del correo electrónico, los foros y la participación activa en proyectos colaborativos utilizando plataformas dela WEB tales como Moodle, Blackboard, o instrumentos de la WEB2.0 tales como las wikis o los blogs, se constituyen en experiencias enriquecedoras del acervo cultural tanto del docente como del estudiante.(Pacheco., 2010)

Según (Ospina B. \& Vasco U., 1994). "El computador es una herramienta de análisis indispensable que facilita el uso de datos reales en clase, que proporciona ilustraciones dinámicas y en gran escala de resultados teóricos en probabilidad y estadística y que sirve como auxiliar de exámenes y encuestas numerosas."

Según el Ministerio de Educación Nacional(MEN, 2003) “Las competencias matemáticas no se alcanzan por generación espontánea, sino que requieren de ambientes de aprendizaje enriquecidos por situaciones problema significativo y comprensivo, que posibiliten avanzar a niveles de competencia más y más complejos."

Esta propuesta de investigación, es el aporte a un proceso estructurado que desarrolla competencias que conducen al estudiante a una apropiación del manejo de las TIC, como apoyo en los procesos educativos y con capacidad para que resuelva problemas específicos de estadística descriptiva; para que sepa diferenciar la información que recibe, igualmente que produzca ideas y sea capaz de comunicarlas a través de la plataforma EDMODO.

El objetivo de la investigación fue implementar Edmodo como una herramienta virtual de aprendizaje en la enseñanza de la estadística descriptiva para grado once de la Institución Educativa General Santander. En exigencia de dar una respuesta a la pregunta de investigación se hace necesario, realizar el desarrollo de varios conceptos para una mayor comprensión, por tal motivo es necesario indagar sobre algunos conceptos:

Las plataformas virtuales educativas son espacios web que ofrecen múltiples servicios a los miembros de la comunidad educativa (profesores, estudiantes, gestores de centros y familias): información, instrumentos para la búsqueda de datos, recursos didácticos, herramientas para la comunicación interpersonal, formación, asesoramiento, entretenimiento. (Bedriñana A, 2005, págs. 81-87).

La mayoría de los portales son gratuitos y constituyen una cortesía de sus patrocinadores (normalmente instituciones administrativas, educativas y empresas del sector) hacia sus destinatarios. De esta manera, mediante la oferta de servicios de interés para los miembros de la comunidad educativa, pretenden difundir una buena imagen institucional o contactar con clientes potenciales. (Marqués G, 2001).

Según (Valverde, 2012).Un aula virtual es un espacio esencialmente diseñado en Internet en el cual profesores y estudiantes interactúan con el fin de realizar actividades que permitan la obtención de aprendizajes aprovechando los recursos disponibles en la red, habitualmente 
asociándose un curso, asignatura o un tema específico, en el cual todos los participantes realizan interacciones con el fin de lograr los objetivos propuestos, ya sean estos individuales o grupales.

Las aulas virtuales hoy toman distintas formas y medidas, y hasta son llamadas con distintos nombres. Algunas son sistemas cerrados en los que el profesor tendrá que volcar sus contenidos y limitarse a las opciones que fueron pensadas por los creadores del espacio virtual, para desarrollar su curso. Otras se extienden a lo largo y ancho de la red usando el hipertexto como su mejor aliado para que los alumnos no dejen de visitar y conocer otros recursos en la red relacionados a la clase. (Norma, 2007)

"Cuando nos referimos a un aula virtual estamos pensando en un conjunto de actividades con un alto ingrediente comunicativo que de otro modo, es decir, sin la experiencia del ordenador conectado a la red, no tendrían el sentido que adquiere en el contexto autentico que proporciona la tecnología”. (Barbera, 2005).

La educación a distancia es una modalidad educativa que permite el acto educativo mediante diferentes métodos, técnicas, estrategias y medios, en una situación en que alumnos y profesores se encuentran separados físicamente y sólo se relacionan de manera presencial ocasionalmente, según sea la distancia, el número de alumnos, tipo de conocimientos que se imparte, etc.(Betancourt, 1993).

Según Ospina Pineda es un entorno de aprendizaje mediado por tecnología, lo cual transforma la relación educativa ya que la acción tecnológica facilita la comunicación y el procesamiento, la gestión y la distribución de la información, agregando a la relación educativa, nuevas posibilidades y limitaciones para el aprendizaje. (Pineda, 2008)

Según (López., 2002). Es que una integración de medios como texto, gráficos, sonidos, animación y video, o los vínculos electrónicos, no tienen sentido sin las dimensiones pedagógicas que el diseñador del ambiente puede darles. Sin embargo, el ambiente de aprendizaje se logra ya en el proceso, cuando estudiantes y docentes (facilitadores), así como los materiales y recursos de información se encuentran interactuando.

Según (Ministerio de Educación. Buenos Aires, 2012, pág. 5) Edmodo es una red social especialmente diseñada para qué estudiantes y docentes pueden intercambiar información, archivos y links en un entorno seguro.Edmodo se puede utilizar en el aula para:

Crear grupos para cada curso con privilegios de profesor para el envío de tareas. Esto permite manejar el intercambio de archivos (fotos - documentos) en un entorno seguro. Ampliar a otras personas, como la familia de los alumnos, algunas tareas o actividades, sin necesidad que sean miembros.

Según (Martin S.2012) “Edmodo pone énfasis en la comunicación y el intercambio de contenidos entre sus usuarios, factor clave para desarrollar su competencia comunicativa. Creada por los estadounidenses Nic Borg y Jeff O'Hara, nació hace cuatro años con el propósito de mejorar el entorno de las escuelas de los distritos y reflejar la conectividad del mundo actual. 


\section{Metodología de la investigación}

La investigación cualitativa es una actividad sistemática orientada a la compresión en profundidad de fenómenos educativos y sociales, a la transformación de prácticas y escenarios. (Sandín E., 2003)

Ahora La investigación cuantitativa es aquella en la que se recogen y analizan datos cuantitativos sobre variables. La investigación cualitativa evita la cuantificación. Los investigadores cualitativos hacen registros narrativos de los fenómenos que son estudiados mediante técnicas como la observación participante y las entrevistas no estructuradas.

\section{Población}

Este proyecto se centra en estudiantes de la jornada de la tarde, específicamente con estudiantes grado once cuyas edades oscilan entre los 15 y 20 años de edad.

\section{Muestra}

La muestra está representada en los estudiantes del grado 1106 a quienes se les aplico la encuesta diagnóstico.

\section{Instrumentos de Investigación}

Para la investigación se utilizaron encuestas directas de carácter individual y de acuerdo a su forma de tipo cerrada. Estás fueron aplicadas como una técnica que utiliza un cuestionario con diversos tipos de preguntas con el fin de conseguir mediciones cuantitativas sobre algunas características objetivas y subjetivas de la población. Estas encuestas serán aplicadas al comienzo de la investigación y al finalizar; está estructurado en tres fases, la primera de diagnóstico, la segunda de diseño y la tercera de verificación.

En una primera fase de la investigación se aplica a 30 estudiantes una primera encuesta para diagnosticar los conocimientos en tecnología y otra sección sobre el uso pedagógico y aulas virtuales. (Ver tabla 1).

En la segunda fase se crea el grupo skymath, en la plataforma edmodo.com, los estudiantes interesados en participar en el aula virtual, deberán registrarse con un código que el docente les asigna y hacer el respectivo registro en la plataforma edmodo.com. Posteriormente ya registrados encontrarán las actividades a desarrollar en la plataforma, además de material de consulta para la asignatura estadística descriptiva. En la actualidad el grupo está conformado por 30 estudiantes del grado 1106 de la JT.

Según (Huamán V., 2005) La encuesta es una técnica destinada a obtener datos de varias personas cuyas opiniones impersonales interesan al investigador. Para ello, a diferencia de la entrevista, se utiliza un listado de preguntas escritas que se entregan a los sujetos, a fin de que las contesten igualmente por escrito. Ese listado se denomina cuestionario. 
Es impersonal porque el cuestionario no lleve el nombre ni otra identificación de la persona que lo responde, ya que no interesan esos datos. Es una técnica que se puede aplicar a sectores más amplios del universo, de manera mucho más económica que mediante entrevistas.

\section{Encuesta aplicada a estudiantes}

Se llevó a cabo una encuesta el día 16 de octubre de 2012, a 30 estudiantes de grado 1106 JT, de la I.E. General Santander. Con un formato impreso, ver anexo 2. Las convenciones utilizadas fueron E: Estudiante, P: Pregunta, como los estudiantes encuestados fueron 30 la tabla 1, y las preguntas desde P1 hasta P7, y la opción de respuestas son SI (S) y NO (N)

P1: Tiene computador en su casa.

$\mathrm{P} 2$.Tiene acceso a Internet en su casa.

P3. Conoce usted que son las TICS.

P4. Utilizando Internet es más fácil elaborar actividades académicas

P5. Conoce usted que es un aula virtual:

P6. Ha realizado formación académica en plataforma virtual

P7. El uso de un aula virtual podría facilitar su aprendizaje.

\section{Tabla 1. Resultados Encuesta Diagnóstica}

\begin{tabular}{lllll}
\hline PREGUNTA & SI & $\%$ & NO & $\%$ \\
\hline Tiene computador en su casa. & 24 & 80 & 6 & 20 \\
Tiene acceso a internet en su casa. & 18 & 60 & 12 & 40 \\
Conoce usted que son las Tics. & 24 & 80 & 6 & 20 \\
Utilizando Internet ha mejorado su rendimiento académico. & 17 & 56.6 & 13 & 43.4 \\
Conoce usted que es un aula virtual. & 22 & 73.3 & 8 & 26.7 \\
Ha realizado formación académica en plataformas virtuales. & 15 & 50 & 15 & 50 \\
El uso de un aula virtual podría facilitar su aprendizaje. & 20 & 66.6 & 10 & 33.4 \\
\hline
\end{tabular}

Pregunta 1: Se observa que el $80 \%$ de los estudiantes tiene computador en su casa, y un $20 \%$ no cuenta con esta herramienta. Lo cual le permite usar esta herramienta en su formación académica.

Pregunta 2: El 60\% de los estudiantes afirman tener acceso a internet y un $40 \%$ no cuentan con este servicio. Por tal motivo es factible que el estudiante pueda ingresar a la plataforma edmodo.com

Pregunta 3: Se observó en la tabla que el $80 \%$ de los estudiantes encuestados conocen que son las tecnologías de la información y la comunicación Tics, y un 20\% no conocen nada al respecto. De tal forma que la gran mayoría de estudiantes se encuentran familiarizados con este tipo de tecnologías en su quehacer diario.

Pregunta 4: Se observó que el 56.4\% de la población encuestada menciona que su rendimiento académico ha mejorado cuando ha utilizado internet, y un $43.4 \%$ manifiesta lo contrario. De esta forma el estudiante afirma que utilizar internet para búsqueda de trabajos en el desarrollo de actividades académicas le ha sido muy útil.

Pregunta 5: El $73.3 \%$ de la población conoce que es un aula virtual y un $26.7 \%$ afirma lo contrario. De esta manera la mayoría de la población encuestada ha tenido contacto con algún tipo de aula virtual, lo cual es relevante para aplicación en la institución educativa.

Pregunta 6: Este ítem presenta una paridad en los resultados ya que el 50\% de estudiantes han tenido formación académica en aulas virtuales y el otro $50 \%$ no. Esto como un modelo 
pedagógico que oriente las acciones para desarrollar la autonomía en los estudiantes y la necesidad de explorar nuevas formas de aprovechar este recurso tecnológico.

El grupo que no posee información acerca del tema lógicamente no está excluido de la formación virtual, para esto se deberá realizar una capacitación sobre el uso y manejo de la plataforma.

Pregunta 7: Se observó que el 66.6\% de los encuestados afirman que el usar un aula virtual podría facilitar su aprendizaje y un 33.4 afirman lo contrario. Un buen porcentaje de estudiantes parece haber observado mejoría en sus habilidades para comunicarse y para manejar la tecnología.

No obstante, los resultados también estarían indicando que el aula virtual podría utilizarse para reducir las horas presenciales sin afectar de manera significativa la adquisición de conocimientos por parte de los estudiantes. Esta última mirada ofrece. Encuesta de satisfacción

Se llevó a cabo una encuesta de satisfacción el día 9 de noviembre de 2012, a 8 estudiantes de grado 1106 JT, de la I.E. General Santander. Con un formato impreso, Ver Tabla 2. Los resultados que obtenidos a través de la encuesta fueron los siguientes: Califique de 1 a 5 los siguientes aspectos del grupo siendo 1 muy difícil, 2 difícil, 3 regular, 4, fácil y 5 muy fácil.

Tabla 2. Resultados encuesta de satisfacción

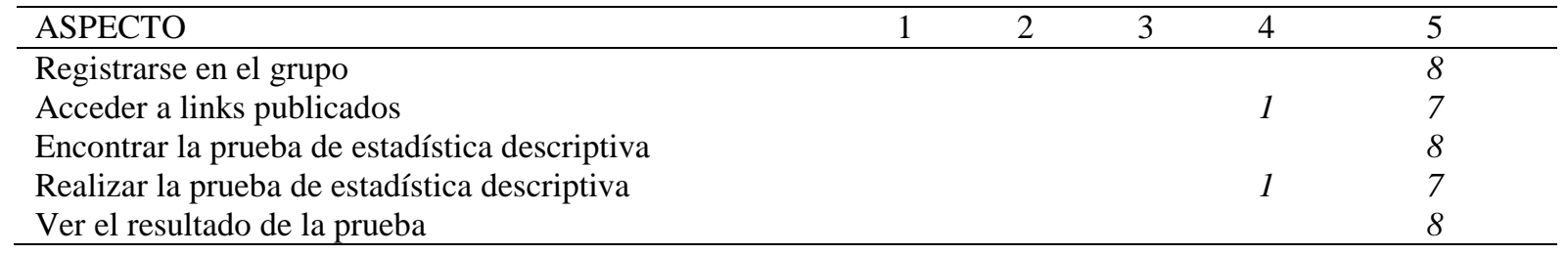

Pregunta 1: Se observó en la tabla que el 100\% de los estudiantes encuestados califico con 5 el registro en la página de edmodo.com, es decir según la escala cualitativa fue muy fácil el ingreso.

Pregunta 2: La tabla nos mostró que el $87.5 \%$ de los estudiantes encuestados califica con un 5 (Muy fácil) ingresar a links publicados en la plataforma como videos, documentos en PDF; y un $12.5 \%$ califican con un 4 (Fácil) encontrar los mismos links.

Pregunta 3: Analizando los datos de la tabla se concluye que el 100\% de los estudiantes encuestados califica con un 5(Muy fácil) encontrar en la página web la prueba de estadística descriptiva.

Pregunta 4: Se observó en la tabla que el $87.5 \%$ de los estudiantes encuestados calificaron con un 5 (Muy fácil) realizar la prueba de estadística en la plataforma. El 12.5\% de los encuestados calificaron con un 4 (Fácil) realizar la prueba en la plataforma.

Pregunta 5: Analizamos en la tabla que el 100\% de los estudiantes encuestados calificaron con un 5 (Muy fácil) ver el resultado de la prueba, que la misma plataforma les da al terminar la prueba.

\section{Conclusiones}

Se pudo implementar en la I.E. General Santander la plataforma virtual Edmodo. En este caso específico en la enseñanza de la estadística descriptiva relacionado con las medidas de 
tendencia central. Las Tecnologías de Información y Comunicaciones (TIC) favorecieron una nueva forma de aprendizaje, en donde se generan espacios virtuales que facilitan interacciones sociales entre los participantes de estos procesos educativos.

Se desarrollaron y diseñaron actividades de enseñanza-aprendizaje, utilizando la plataforma Edmodo en la asignatura estadística descriptiva. Se evaluó la aceptación de Edmodo, observando un gran beneplácito de la plataforma por parte de los estudiantes de grado 1106 de la institución educativa General Santander.

\section{Bibliografía}

Betancourt, A. M. (Mayo de 1993). La educación a distancia y la función tutorial. Obtenido de http://www.usc.es/ceta/recursos/documentos/tutoria.pdf

Condie, R. y. (2007). The impact of ICT - a landscape review. London: Becta Research.

Huamán, H. (2005). Manual de Técnicas de Investigación. Conceptos y aplicaciones. Perú.

López, R. (2002). Presimposio Virtual Somece 2002. Obtenido de http://www.informaticaeducativa.com/virtual2002/mesas/uno/ava.pdf

Marqués. P. (2001). Evaluación De Los Portales Educativos En Internet. Revista Pixel. Bit, 18.

Men, M. D. (Mayo De 2003). Estándares Básicos De Competencias En Matemáticas. Obtenido De Http://Www.Eduteka.Org/Pdfdir/Menestandaresmatematicas2003.Pdf

Ministerio De Educación. Buenos Aires. (19 de Marzo de 2012). Tutorial de Edmodo. Obtenido de http://integrar.bue.edu.ar/wp-content/uploads/2012/03/Tutorial_Edmodo.pdf

Norma, S. (2007). El aula virtual: usos y elementos que la componen.

Orellana, L. (Marzo de 2001). Estadística Descriptiva. Obtenido de http://www.hacienda.go.cr/cifh/sidovih/cursos/material_de_apoyo-F-C-

CIFH/1MaterialdeapoyocursosCIFH/4Estad\%C3\%ADsticaBasica/EstadisticadescriptivaLillianaOrellana.pdf

Ospina Botero., D., \& Vasco Uribe., C. (1994). Congresos Internacionales Sobre La Enseñanza P E La Estadística: Una Vision Histórica Y De Contenido. Obtenido De Revistas.Unal.Edu.Co/Index.Php/Estad/Article/.../10542

Pacheco. P. (2010). Nuevas competencias docentes en la enseñanza de la probabilidad y estadística. Bogotá: Universidad Nacional de Colombia.

Pineda. P. (2008). ¿Qué Es Un Ambiente Virtual De Aprendizaje? Obtenido http://aprendeenlinea.udea.edu.co/banco/html/ambiente_virtual_deprendizaje/

Sandín. P. (2003). Investigación cualitativa en investigación. Fundamentos y tradiciones. Madrid: McGgraw-Hill.

Unesco. (2005). Informe De Seguimiento De La Educación Para Todos En El Mundo 2005. París: Unesco. 